\title{
CUBIC RANK TRANSMUTED MODIFIED BURR III DISTRIBUTION: DEVELOPMENT, PROPERTIES, CHARACTERIZATIONS AND APPLICATIONS
}

\author{
Fiaz Ahmad Bhatti ${ }^{1}$, G.G. Hamedani ${ }^{2}$, Seyed Morteza Najibi ${ }^{3}$ and Munir Ahmad ${ }^{1}$ \\ ${ }^{1}$ National College of Business Administration and Economics \\ ${ }^{2}$ Marquette University, Milwaukee \\ ${ }^{3}$ Department of Statistics, College of Sciences, Shiraz University
}

\begin{abstract}
We propose a lifetime distribution with flexible hazard rate called cubic rank transmuted modified Burr III (CRTMBIII) distribution. We develop the proposed distribution on the basis of the cubic ranking transmutation map. The density function of CRTMBIII is symmetrical, right-skewed, left-skewed, exponential, arc, $\mathrm{J}$ and bimodal shaped. The flexible hazard rate of the proposed model can accommodate almost all types of shapes such as unimodal, bimodal, arc, increasing, decreasing, decreasing-increasing-decreasing, inverted bathtub and modified bathtub. To show the importance of proposed model, we present mathematical properties such as moments, incomplete moments, inequality measures, residual life function and stress strength reliability measure. We characterize the CRTMBIII distribution via techniques. We address the maximum likelihood method for the model parameters. We evaluate the performance of the maximum likelihood estimates (MLEs) via simulation study. We establish empirically that the proposed model is suitable for strengths of glass fibers. We apply goodness of fit statistics and the graphical tools to examine the potentiality and utility of the CRTMBIII distribution.
\end{abstract}

Keywords: Moments, Reliability, Characterizations, Maximum Likelihood Estimation. 


\section{INTRODUCTION}

In recent decades, many continuous univariate distributions have been developed, however, various data sets from reliability, insurance, finance, climatology, biomedical sciences and other areas do not follow these distributions. Therefore, modified, extended and generalized distributions and their applications to problems in these areas is a clear need of day.

The modified, extended and generalized distributions are obtained by the introduction of some transformation or addition of one or more parameters to the well-known baseline distributions. These new developed distributions provide better fit to the data than the sub and competing models.

Shaw and Buckley (2009) proposed ranking quadratic transmutation map to solve financial problems.

\subsection{Quadratic Ranking Transmutation Map}

Theorem 1.1: Let $X_{1}$ and $X_{2}$ be independent and identically distributed (i.i.d.) random variables with the common cumulative distribution function $G(x)$. Then, the ranking quadratic transmutation map is

$$
F_{Y}(x)=(1+\lambda) G(x)-\lambda G^{2}(x), \lambda \in[-1,1] .
$$

\section{Proof}

Let $X_{1}$ and $X_{2}$ be i.i.d. random variables with the common cumulative distribution function $G(x)$. Now, consider the following order statistics:

$$
X_{1: 2}=\min \left(X_{1}, X_{2}\right) \text { and } X_{2: 2}=\max \left(X_{1}, X_{2}\right)
$$

let $Y \stackrel{d}{=} X_{1: 2}$, with probability $\pi, \stackrel{d}{=} X_{2: 2}$, with probability $1-\pi$, where $0 \leq \pi \leq 1$. The cumulative distribution function of $\mathrm{Y}$ is

$$
F_{Y}(x)=\pi \mathrm{P}\left(X_{1: 2} \leq x\right)+(1-\pi) \operatorname{Pr}\left(X_{2: 2} \leq x\right),
$$

or

$$
\begin{gathered}
F_{Y}(x)=\pi\left[1-[1-G(x)]^{2}\right]+(1-\pi)[G(x)]^{2}, \\
F_{Y}(x)=2 \pi G(x)+(1-2 \pi)[G(x)]^{2} .
\end{gathered}
$$

If we take $\lambda=2 \pi-1$, the distribution in equation (2) is known as ranking quadratic transmutation map or transmuted distribution.

\subsection{Cubic Ranking Transmutation Map}

Theorem 1.1: Let $X_{1}, X_{2}$ and $X_{3}$ be i.i.d. random variables with the common cumulative distribution function $G(z)$. Then, the cubic ranking transmutation map is

\section{Proof}

$$
F(x)=\lambda_{1} G(x)+\left(\lambda_{2}-\lambda_{1}\right) G^{2}(x)+\left(1-\lambda_{2}\right) G^{3}(x), \lambda_{1} \in[0,1], \lambda_{2} \in[-1,1] .
$$


Consider the following order statistics:

$X_{1: 3}=\min \left(X_{1}, X_{2}, X_{3}\right), X_{2: 3}$ and $X_{3: 3}=\max \left(X_{1}, X_{2}, X_{3}\right)$.

Let $Y \stackrel{d}{=} X_{1: 3}$, with probability $\pi_{1}, Y \stackrel{d}{=} X_{2: 3}$, with probability $\pi_{2}, Y \stackrel{d}{=} X_{3: 3}$, with probability $\pi_{3}$, where $0 \leq \pi_{i} \leq 1, \pi_{3}=1-\pi_{1}-\pi_{2}$ and $\sum_{i=1}^{3} \pi_{i}=1$.

The cumulative distribution function (cdf) of $\mathrm{Y}$ is

$$
F_{Y}(x)=\pi_{1} \mathrm{P}\left(X_{1: 3} \leq x\right)+\pi_{2} \mathrm{P}\left(X_{2: 3} \leq x\right)+\left(1-\pi_{1}-\pi_{2}\right) \mathrm{P}\left(X_{3: 3} \leq x\right),
$$

where $\mathrm{P}\left(X_{1: 3} \leq x\right)=1-[1-G(x)]^{3}, \operatorname{Pr}\left(X_{2 ; 3} \leq x\right)=3 G^{2}(x)-2 G^{3}(x)$ and $\mathrm{P}\left(X_{3: 3} \leq x\right)=[G(x)]^{3}$.

Now, the cdf of $Y$ becomes

$$
F_{Y}(x)=3 \pi_{1} G(x)+3\left(\pi_{2}-\pi_{1}\right)[G(x)]^{2}+\left(1-\pi_{2}\right)[G(x)]^{3} .
$$

If we take $\lambda_{1}=3 \pi_{1}$ and $\lambda_{2}=3 \pi_{2}$ the distribution in equation (4) is known as cubic ranking transmutation map or transmuted distribution of order 2.

Definition 1.1: The cdf and probability density function (pdf) for cubic rank transmuted distribution are given, respectively, by

$$
F(x)=\lambda_{1} G(x)+\left(\lambda_{2}-\lambda_{1}\right) G^{2}(x)+\left(1-\lambda_{2}\right) G^{3}(x), \lambda_{1} \in[0,1], \lambda_{2} \in[-1,1],
$$

and

$$
f(x)=g(x)\left[\lambda_{1}+2\left(\lambda_{2}-\lambda_{1}\right) G(x)+3\left(1-\lambda_{2}\right) G^{2}(x)\right], x \in R
$$

Afify et al. (2017) proposed the beta transmuted-H family of distributions. Al-Kadim and Mohammed (2017) presented the cubic transmuted Weibull distribution in terms of basic mathematical properties. Nofal et al. (2017) studied a generalized transmuted-G family of distributions. Alizadeh et al. (2017) developed generalized transmuted family of distributions. Bakouch et al. (2017) also introduced a new family of transmuted distributions. Granzotto et al. (2017) proposed a cubic ranking transmutation map and its studied different properties. They studied properties of cubic rank transmuted Weibull distribution and cubic rank transmuted log logistic distribution. Yilmaz (2018) proposed a new distribution family developed with polynomial rank transmutation. Rahman et al. (2018) developed general family of transmuted distributions. Riffi (2019) presented higher rank transmuted families of distributions. Rahman et al. (2019) studied properties of cubic rank transmuted Weibull distribution.

Burr III (Burr; 1942) has wide range of applications in failure time modeling, reliability, business failure data, modeling finance, insurance data and quality control plans. Burr III (BIII) model accommodates only decreasing and inverted bathtub hazard rate functions (hrf). Transmuted Burr III (TBIII) accommodates only inverted bathtub hazard rate functions (Abdul-Moniem; 2015). The failure rate for modified Burr III (MBIII) can take only increasing, decreasing, inverted bathtub and modified bathtub shapes (Bhatti et al. 2019). Transmuted modified Burr III (TMBIII) accommodates only decreasing and inverted bathtub hazard rate functions (Ali and Ahmad; 2016). The hrf for the CRTMBIII distribution accommodates almost all shapes such as bimodal, arc, increasing, decreasing, decreasing- 
increasing- decreasing, inverted bathtub (unimodal) and modified bathtub. Due to its flexible failure rate, it can be applicable to lifetime applications.

The basic motivations for proposing the CRTMBIII distribution are: (i) to generate distributions with symmetrical, right-skewed, left-skewed, exponential, arc, J and bimodal shaped; (ii) to obtain unimodal, bimodal, arc, increasing, decreasing, decreasing-increasingdecreasing, inverted bathtub and modified bathtub hazard rate function; (iii) to serve as the best alternative model for the current models to explore and modeling real data in economics, life testing, reliability, survival analysis manufacturing and other areas of research and (iv) to provide better fits than other sub-models.

This paper is sketched into the following sections. In Section 2, we develop and study the CRTMBIII distribution. We also present the basic structural properties and sub-models. We also study some plots of density and hazard rate functions. In Section 3, we derive mathematical properties such as moments, incomplete moments, inequality measures, residual and reverse residual life function and stress-strength reliability measure. In Section 4, two characterizations of the CRTMBIII distribution are studied. In Section 5, we address the parameters of the CRTMBIII distribution via maximum likelihood method. In Section 6, we evaluate the performance of the maximum likelihood estimates (MLEs) of the modal parameters via simulation study. In Section 7, we establish empirically that the proposed model is suitable for strengths of glass fibers. We apply goodness of fit statistics and graphical tools to examine the potentiality and utility of the CRTMBIII distribution. The concluding remarks are given in Section 8.

\section{THE CRTMBIII DISTRIBUTION}

Ali et al. (2015) studied modified Burr III (MBIII) distribution with its properties. Ali and Ahmad (2015) studied transmuted MBIII (TMBIII) distribution and its properties. The cdf and pdf of MBIII distribution are given, respectively, by

$$
F(x)=\left(1+\gamma x^{-\beta}\right)^{-\frac{\alpha}{\gamma}}, x \geq 0,
$$

and

$$
f(x)=\alpha \beta x^{-\beta-1}\left(1+\gamma x^{-\beta}\right)^{-\frac{\alpha}{\gamma}-1} \quad x>0, \alpha>0, \beta>0, \gamma>0 .
$$

Here, the CRTMBIII distribution is introduced with the help of (7) and (8). The cdf and pdf of the CRTMBIII distribution are given, respectively, by

$$
F(x)=\lambda_{1}\left(1+\gamma x^{-\beta}\right)^{-\frac{\alpha}{\gamma}}+\left(\lambda_{2}-\lambda_{1}\right)\left(1+\gamma x^{-\beta}\right)^{-\frac{2 \alpha}{\gamma}}+\left(1-\lambda_{2}\right)\left(1+\gamma x^{-\beta}\right)^{-\frac{3 \alpha}{\gamma}}, \quad x \geq 0,
$$

and

$$
f(x)=\alpha \beta x^{-\beta-1}\left[\lambda_{1}\left(1+\gamma x^{-\beta}\right)^{-\frac{\alpha}{\gamma}-1}+2\left(\lambda_{2}-\lambda_{1}\right)\left(1+\gamma x^{-\beta}\right)^{-\frac{2 \alpha}{\gamma}-1}+3\left(1-\lambda_{2}\right)\left(1+\gamma x^{-\beta}\right)^{-\frac{3 \alpha}{\gamma}-1}\right], x>0,
$$

with $\alpha>0, \beta>0, \gamma>0, \lambda_{1} \in[0,1], \lambda_{2} \in[-1,1]$. 
In future, the pdf in (11) is denoted by X CRTMBIII $\left(\alpha, \beta, \gamma, \lambda_{1}, \lambda_{2}\right)$.

\subsection{Structural Properties}

For X CRTMBIII $\left(\alpha, \beta, \gamma, \lambda_{1}, \lambda_{2}\right)$, the survival, hazard, cumulative hazard, reverse hazard functions and the Mills ratio are given, respectively, by

$$
\begin{gathered}
S(x)=1-\left[\lambda_{1}\left(1+\gamma x^{-\beta}\right)^{-\frac{\alpha}{\gamma}}+\left(\lambda_{2}-\lambda_{1}\right)\left(1+\gamma x^{-\beta}\right)^{-\frac{2 \alpha}{\gamma}}+\left(1-\lambda_{2}\right)\left(1+\gamma x^{-\beta}\right)^{-\frac{3 \alpha}{\gamma}}\right], x \geq 0, \\
h(x)=\frac{\alpha \beta x^{-\beta-1}\left[\lambda_{1}\left(1+\gamma x^{-\beta}\right)^{-\frac{\alpha}{\gamma}-1}+2\left(\lambda_{2}-\lambda_{1}\right)\left(1+\gamma x^{-\beta}\right)^{-\frac{2 \alpha}{\gamma}-1}+3\left(1-\lambda_{2}\right)\left(1+\gamma x^{-\beta}\right)^{-\frac{3 \alpha}{\gamma}-1}\right]}{1-\left[\lambda_{1}\left(1+\gamma x^{-\beta}\right)^{-\frac{\alpha}{\gamma}}+\left(\lambda_{2}-\lambda_{1}\right)\left(1+\gamma x^{-\beta}\right)^{-\frac{2 \alpha}{\gamma}}+\left(1-\lambda_{2}\right)\left(1+\gamma x^{-\beta}\right)^{-\frac{3 \alpha}{\gamma}}\right]}, \\
r(x)=\alpha \beta x^{-\beta-1}\left(1+\gamma x^{-\beta}\right)^{-1}\left[\frac{\lambda_{1}+2\left(\lambda_{2}-\lambda_{1}\right)\left(1+\gamma x^{-\beta}\right)^{-\frac{\alpha}{\gamma}}+3\left(1-\lambda_{2}\right)\left(1+\gamma x^{-\beta}\right)^{-\frac{2 \alpha}{\gamma}}}{\left.\lambda_{1}+\left(\lambda_{2}-\lambda_{1}\right)\left(1+\gamma x^{-\beta}\right)^{-\frac{\alpha}{\gamma}}+\left(1-\lambda_{2}\right)\left(1+\gamma x^{-\beta}\right)^{-\frac{2 \alpha}{\gamma}}\right],}\right] \\
H(x)=-\ln \left\{1-\left[\lambda_{1}\left(1+\gamma x^{-\beta}\right)^{-\frac{\alpha}{\gamma}}+\left(\lambda_{2}-\lambda_{1}\right)\left(1+\gamma x^{-\beta}\right)^{-\frac{2 \alpha}{\gamma}}+\left(1-\lambda_{2}\right)\left(1+\gamma x^{-\beta}\right)^{-\frac{3 \alpha}{\gamma}}\right]\right\},
\end{gathered}
$$

and

$$
m(x)=\frac{1-\left[\lambda_{1}\left(1+\gamma x^{-\beta}\right)^{-\frac{\alpha}{\gamma}}+\left(\lambda_{2}-\lambda_{1}\right)\left(1+\gamma x^{-\beta}\right)^{-\frac{2 \alpha}{\gamma}}+\left(1-\lambda_{2}\right)\left(1+\gamma x^{-\beta}\right)^{-\frac{3 \alpha}{\gamma}}\right]}{\alpha \beta x^{-\beta-1}\left[\lambda_{1}\left(1+\gamma x^{-\beta}\right)^{-\frac{\alpha}{\gamma}-1}+2\left(\lambda_{2}-\lambda_{1}\right)\left(1+\gamma x^{-\beta}\right)^{-\frac{2 \alpha}{\gamma}-1}+3\left(1-\lambda_{2}\right)\left(1+\gamma x^{-\beta}\right)^{-\frac{3 \alpha}{\gamma}-1}\right]} .
$$

The elasticity $e(x)=\frac{d \ln F(x)}{d \ln x}=x r(x)$ for the CRTMBIII distribution is

The elasticity of the CRTMBIII distribution shows the behavior of the accumulation of probability in the domain of the random variable.

The quantile function of the CRTMBIII distribution is the solution of the following

$$
x_{q}=\left[\frac{1}{\gamma}\left(-\frac{B}{3 A}-\frac{2^{1 / 3}\left(-B^{2}+3 A C\right)}{3 A M}+\frac{M}{32^{1 / 3} A}\right)^{-\frac{\gamma}{\alpha}}-\frac{1}{\gamma}\right]^{-\frac{1}{\beta}},
$$

where $A=\left(1-\lambda_{2}\right), B=\left(\lambda_{2}-\lambda_{1}\right), C=\lambda_{1}$ and

$$
M=\left[-2 B^{3}+9 A B C+27 A^{2} q+\sqrt{4\left(-B^{2}+3 A C\right)^{3}+\left(-2 B^{3}+9 A B C+27 A^{2} q\right)^{2}}\right]^{1 / 3}
$$


The random number generator of the CRTMBIII distribution is the solution of the following

$$
X=\left[\frac{1}{\gamma}\left(-\frac{B}{3 A}-\frac{2^{1 / 3}\left(-B^{2}+3 A C\right)}{3 A \mathrm{M}_{Z}}+\frac{\mathrm{M}_{Z}}{32^{1 / 3} A}\right)^{-\frac{\gamma}{\alpha}}-\frac{1}{\gamma}\right]^{-\frac{1}{\beta}},
$$

where $\mathrm{M}_{Z}=\left[-2 B^{3}+9 A B C+27 A^{2} Z+\sqrt{4\left(-B^{2}+3 A C\right)^{3}+\left(-2 B^{3}+9 A B C+27 A^{2} Z\right)^{2}}\right]^{1 / 3}$ and the random variable $\mathrm{Z}$ has the uniform distribution on $(0,1)$.

\subsection{Shapes of the CRTMBIII Density and Hazard Rate Functions}

The following graphs show that shapes of CRTMBIII density are arc, exponential, positively skewed, negatively skewed and symmetrical (Fig.1). The CRTMBIII distribution has unimodal, bimodal, arc, increasing, decreasing, decreasing-increasing- decreasing, inverted bathtub and modified bathtub hazard rate function (Fig. 2).

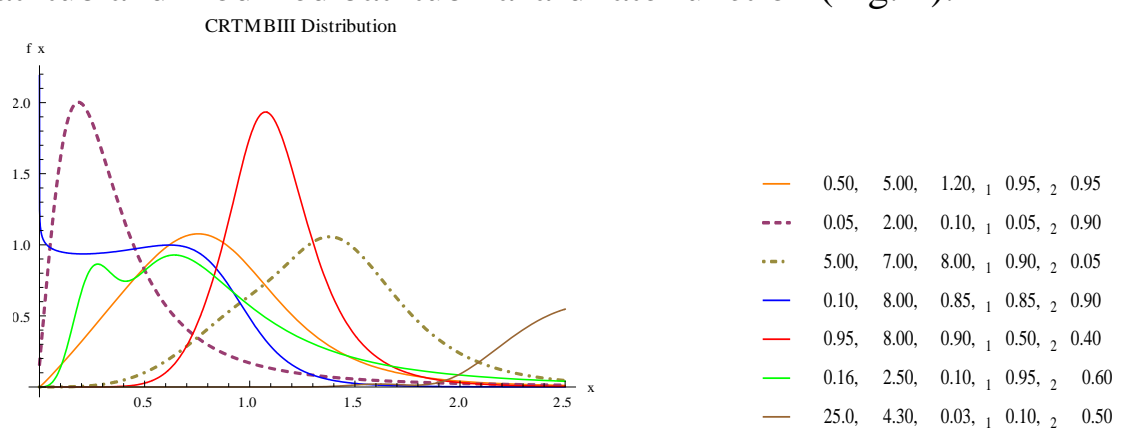

Fig.1 Plots of pdf of the CRTMBIII distribution for the selected parameter values

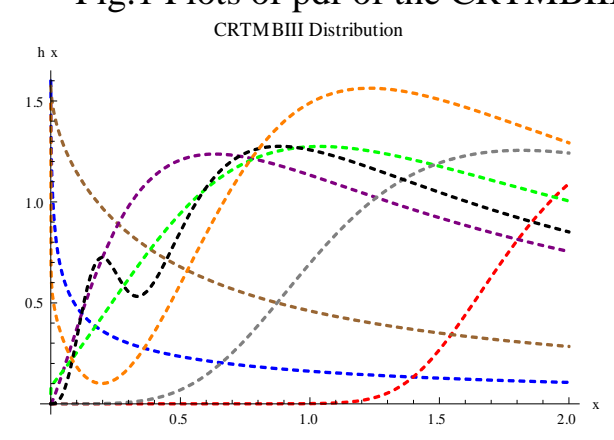

$\begin{array}{llllll}- & 1.00, & 0.50, & 0.50,{ }_{1} & 0.40,{ }_{2} & 0.60 \\ - & 11.0, & 4.35, & 1.50,{ }_{1} & 0.04,{ }_{2} & 0.70 \\ - & 0.50, & 0.80, & 0.40,{ }_{1} & 0.50,{ }_{2} & 0.90 \\ - & 0.50, & 1.75, & 0.40,{ }_{1} & 0.90,{ }_{2} & 0.95 \\ - & 0.40, & 2.35, & 0.90,{ }_{1} & 0.10,{ }_{2} & 0.90 \\ - & 3.10, & 3.25, & 1.80,{ }_{1} & 0.95, & 0.95 \\ - & 0.20, & 1.90, & 0.10,{ }_{1} & 0.80,{ }_{2} & 0.90 \\ - & 0.27, & 2.90, & 0.85,{ }_{1} & 0.40, & 0.95\end{array}$

Fig:2 Plots of hrf of the CRTMBIII distribution for the selected parameter values

\subsection{Sub-Models}

The CRTMBIII distribution has the following sub models (Table 1). 
Table 1: Sub-Models of the CRTMBIII Distribution

\begin{tabular}{|l|l|l|l|l|l|l|l|}
\hline Sr.No. & $\mathrm{X}$ & $\alpha$ & $\beta$ & $\gamma$ & $\lambda_{1}$ & $\lambda_{2}$ & Name of Distribution \\
\hline 1 & $\mathrm{X}$ & $\alpha$ & $\beta$ & $\gamma$ & $\lambda_{1}$ & $\lambda_{2}$ & CRTMBIII distribution \\
\hline 2 & $\mathrm{X}$ & $\alpha$ & $\beta$ & 1 & $\lambda_{1}$ & $\lambda_{2}$ & CRTBIII distribution \\
\hline 3 & $\mathrm{X}$ & 1 & $\beta$ & 1 & $\lambda_{1}$ & $\lambda_{2}$ & CRTLL distribution \\
\hline 4 & $\mathrm{X}$ & $\alpha$ & $\beta$ & $\gamma$ & $\lambda_{1}$ & 1 & TMBIII distribution \\
\hline 5 & $\mathrm{X}$ & $\alpha$ & $\beta$ & 1 & $\lambda_{1}$ & 1 & TBIII distribution \\
\hline 6 & $\mathrm{X}$ & 1 & $\beta$ & 1 & $\lambda_{1}$ & 1 & TLL distribution \\
\hline 7 & $\mathrm{X}$ & $\alpha$ & $\beta$ & $\gamma \rightarrow \infty$ & $\lambda_{1}$ & $\lambda_{2}$ & CRTGIW distribution \\
\hline 8 & $\mathrm{X}$ & $\alpha$ & $\beta$ & $\gamma \rightarrow \infty$ & $\lambda_{1}$ & 1 & TGIW distribution \\
\hline 9 & $\mathrm{X}$ & $\alpha$ & $\beta$ & $\gamma \rightarrow \infty$ & 1 & 1 & GIW distribution \\
\hline 10 & $\mathrm{X}$ & $\alpha$ & $\beta$ & $\gamma$ & 1 & 1 & MBIII distribution \\
\hline 11 & $\mathrm{X}$ & $\alpha$ & $\beta$ & 1 & 1 & 1 & BIII distribution \\
\hline 12 & $\mathrm{X}$ & 1 & $\beta$ & 1 & 1 & 1 & LL distribution \\
\hline
\end{tabular}

\section{MATHEMATICAL PROPERTIES}

We derive theoretically some mathematical properties such as the $\mathrm{r}^{\text {th }}$ ordinary moments, $\mathrm{s}^{\text {th }}$ incomplete moments, and inequality measures, residual and reverse residual life function and reliability measures in this section.

\subsection{Ordinary Moments}

The moments are significant tools for statistical analysis in pragmatic sciences. The descriptive measures such as central tendency $\left(\mu_{1}^{\prime}\right)$, dispersion $(\sigma)$, skewness $\left(\gamma_{1}\right)$ and kurtosis $\left(\gamma_{2}\right)$ can be calculated from the moments.

For X CRTMBIII $\left(\alpha, \beta, \gamma, \lambda_{1}, \lambda_{2}\right)$, the $r$ th ordinary moment is

$$
\begin{gathered}
E\left(X^{r}\right)=\int_{0}^{\infty} x^{r} f(x) d x \\
\mu_{r}^{\prime}=E\left(X^{r}\right)=\int_{0}^{\infty} x^{r} \alpha \beta x^{-\beta-1}\left[\lambda_{1}\left(1+\gamma x^{-\beta}\right)^{-\frac{\alpha}{\gamma}-1}+2\left(\lambda_{2}-\lambda_{1}\right)\left(1+\gamma x^{-\beta}\right)^{-\frac{2 \alpha}{\gamma}-1}\right. \\
\left.+3\left(1-\lambda_{2}\right)\left(1+\gamma x^{-\beta}\right)^{-\frac{3 \alpha}{\gamma}-1}\right] d x .
\end{gathered}
$$

Letting $\gamma x^{-\beta}=y, x=\left(\frac{y}{\gamma}\right)^{-\frac{1}{\beta}}, \gamma \beta x^{-\beta-1} d x=-d y$, then 


$$
\begin{aligned}
\mu_{r}^{\prime}=E\left(X^{r}\right)=[ & \lambda_{1} \frac{\alpha}{\gamma} \gamma^{\frac{r}{\beta}} B\left(1-\frac{r}{\beta}, \frac{\alpha}{\gamma}+\frac{r}{\beta}\right)+2\left(\lambda_{2}-\lambda_{1}\right) \frac{\alpha}{\gamma} \gamma^{\frac{r}{\beta}} B\left(1-\frac{r}{\beta}, \frac{2 \alpha}{\gamma}+\frac{r}{\beta}\right) \\
& \left.+3\left(1-\lambda_{2}\right) \frac{\alpha}{\gamma} \gamma^{\frac{r}{\beta}} B\left(1-\frac{r}{\beta}, \frac{3 \alpha}{\gamma}+\frac{r}{\beta}\right)\right] \\
\mu_{r}^{\prime}=E\left(X^{r}\right)= & \gamma^{\frac{r}{\beta}} \Gamma\left(1-\frac{r}{\beta}\right)\left[\lambda_{1} \frac{\Gamma\left(\frac{\alpha}{\gamma}+\frac{r}{\beta}\right)}{\Gamma\left(\frac{\alpha}{\gamma}\right)}+\left(\lambda_{2}-\lambda_{1}\right) \frac{\Gamma\left(\frac{2 \alpha}{\gamma}+\frac{r}{\beta}\right)}{\Gamma\left(\frac{2 \alpha}{\gamma}\right)}\right. \\
& \left.+\left(1-\lambda_{2}\right) \frac{\Gamma\left(\frac{3 \alpha}{\gamma}+\frac{r}{\beta}\right)}{\Gamma\left(\frac{3 \alpha}{\gamma}\right)}\right], r=1,2,3,4, \ldots,
\end{aligned}
$$

where $\Gamma(.,$.$) is a gamma function.$

Mean and Variance of the CRTMBIII distribution are

$$
\begin{aligned}
& \operatorname{Var}(X)
\end{aligned}
$$

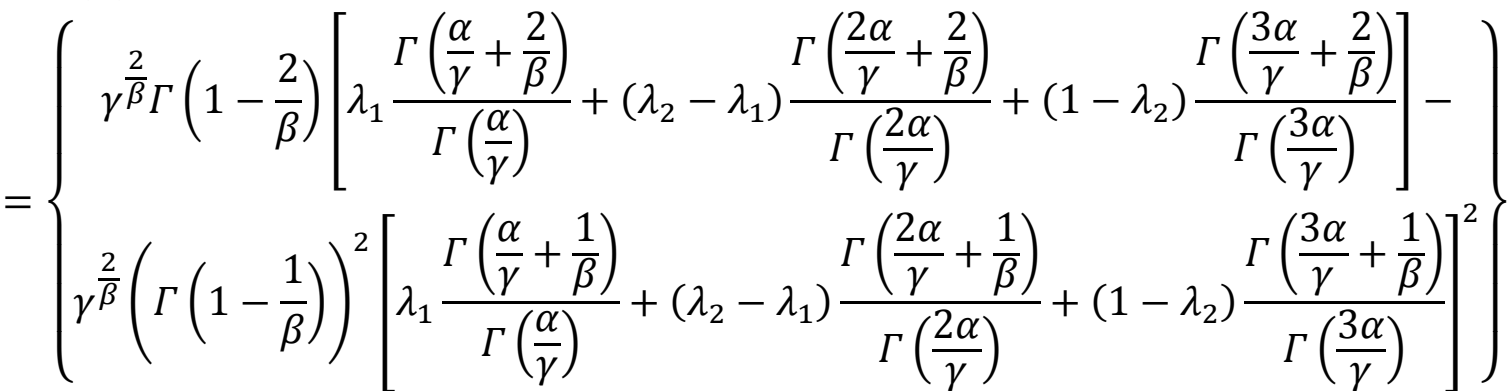$$
E(X)=\gamma^{\frac{1}{\beta}} \Gamma\left(1-\frac{1}{\beta}\right)\left[\lambda_{1} \frac{\Gamma\left(\frac{\alpha}{\gamma}+\frac{1}{\beta}\right)}{\Gamma\left(\frac{\alpha}{\gamma}\right)}+\left(\lambda_{2}-\lambda_{1}\right) \frac{\Gamma\left(\frac{2 \alpha}{\gamma}+\frac{1}{\beta}\right)}{\Gamma\left(\frac{2 \alpha}{\gamma}\right)}+\left(1-\lambda_{2}\right) \frac{\Gamma\left(\frac{3 \alpha}{\gamma}+\frac{1}{\beta}\right)}{\Gamma\left(\frac{3 \alpha}{\gamma}\right)}\right],
$$

The Mellin transformation is applied to get the moments of a probability distribution. For $\mathrm{X} \sim \operatorname{CRTMBIII}\left(\alpha, \beta, \gamma, \lambda_{1}, \lambda_{2}\right)$, the Mellin transform is

$$
\begin{aligned}
& M\{f(x) ; \mathrm{s}\}=f^{*}(s)=E\left(X^{s-1}\right), \\
& \mathrm{M}\{f(x) ; \mathrm{s}\}=\gamma^{\frac{s-1}{\beta}} \Gamma\left(1-\frac{s-1}{\beta}\right)\left[\lambda_{1} \frac{\Gamma\left(\frac{\alpha}{\gamma}+\frac{s-1}{\beta}\right)}{\Gamma\left(\frac{\alpha}{\gamma}\right)}+\left(\lambda_{2}-\lambda_{1}\right) \frac{\Gamma\left(\frac{2 \alpha}{\gamma}+\frac{s-1}{\beta}\right)}{\Gamma\left(\frac{2 \alpha}{\gamma}\right)}+\left(1-\lambda_{2}\right) \frac{\Gamma\left(\frac{3 \alpha}{\gamma}+\frac{s-1}{\beta}\right)}{\Gamma\left(\frac{3 \alpha}{\gamma}\right)}\right] \text {. }
\end{aligned}
$$

The $\mathrm{r}^{\text {th }}$ central moment $\left(\mu_{r}\right)$, coefficients of skewness $\left(\gamma_{1}\right)$ and kurtosis $\left(\gamma_{2}\right)$ for the CRTMBIII model are attained from $\mu_{r}=\sum_{\ell=1}^{r}(-1)^{\ell}\left(\begin{array}{l}r \\ l\end{array}\right) \mu_{\ell}^{\prime} \mu_{r-\ell}^{\prime}, \gamma_{1}=\frac{\mu_{3}}{\left(\mu_{2}\right)^{\frac{3}{2}}}$ and $\beta_{2}=$ $\frac{\mu_{4}}{\left(\mu_{2}\right)^{2}}$. The numerical values for the mean $\left(\mu_{1}^{\prime}\right)$, median $(\tilde{\mu})$, standard deviation $(\sigma)$, skewness $\left(\gamma_{1}\right)$ and kurtosis $\left(\gamma_{2}\right)$ for the CRTMBIII distribution for selected values of $\alpha, \beta, \gamma, \lambda_{1}, \lambda_{2}$ are listed in Table 2. We also depict that the CRTMBIII model can be effective to model data sets in terms of the descriptive measures. 
Table 2: $\mu_{1}^{\prime}, \tilde{\mu}, \sigma, \gamma_{1}$ and $\gamma_{2}$ of the CRTMBIII Distribution

\begin{tabular}{|l|l|l|l|l|r|l|r|r|r|}
\hline$\alpha$ & $\beta$ & $\gamma$ & $\lambda_{1}$ & \multicolumn{1}{l|}{$\lambda_{2}$} & \multicolumn{1}{l|}{$\mu_{1}^{\prime}$} & $\tilde{\mu}$ & \multicolumn{1}{l|}{$\sigma$} & \multicolumn{1}{l|}{$\gamma_{1}$} & \multicolumn{1}{l|}{$\gamma_{2}$} \\
\hline 1.5 & 1.5 & 1 & 0.1 & -0.1 & 7.0946 & 3.2782 & 80.9775 & 521.808 & 374033 \\
\hline 0.5 & 3 & 1.5 & 0.1 & -0.1 & 1.3711 & 1.1392 & 1.114 & 9.149 & 341.352 \\
\hline 0.5 & 5 & 1.5 & 0.1 & -0.1 & 1.1488 & 1.0813 & 0.4703 & 2.2561 & 22.8658 \\
\hline 0.1 & 5 & 1.5 & 0.1 & 0.1 & 0.5778 & 0.5102 & 0.4486 & 1.937 & 35.0744 \\
\hline 0.1 & 5 & 1.5 & 0.5 & 0.5 & 0.4518 & 0.3437 & 0.4419 & 1.9544 & 28.7892 \\
\hline 0.1 & 5 & 1.5 & 0.5 & -0.5 & 0.5822 & 0.525 & 0.4629 & 1.4152 & 11.7208 \\
\hline 0.1 & 5 & 1.5 & 0.9 & -0.5 & 0.508 & 0.4299 & 0.4702 & 1.4098 & 10.0304 \\
\hline 0.1 & 5 & 1.5 & 0.9 & 0.5 & 0.3785 & 0.2292 & 0.4267 & 1.7812 & 11.2591 \\
\hline 0.1 & 5 & 1.5 & 0.9 & 0.9 & 0.326 & 0.1683 & 0.397 & 2.3369 & 2.3369 \\
\hline 0.5 & 10 & 1 & 0.5 & 0.5 & 0.9873 & 0.9859 & 0.2186 & 0.3888 & 5.8513 \\
\hline 0.05 & 10 & 1 & 0.5 & 0.5 & 0.4815 & 0.4646 & 0.3378 & 0.3436 & 2.397 \\
\hline 0.05 & 10 & 3 & 0.5 & 0.5 & 0.2712 & 0.1121 & 0.3353 & 1.3062 & 3.9946 \\
\hline 0.05 & 10 & 3 & 0.1 & 0.1 & 0.3586 & 0.2449 & 0.3523 & 0.9644 & 3.1294 \\
\hline 0.05 & 10 & 0.5 & 0.1 & 0.1 & 0.7113 & 0.7306 & 0.2515 & -0.0875 & 3.7189 \\
\hline 0.05 & 10 & 0.8 & 0.1 & 0.1 & 0.6368 & 0.6537 & 0.293 & 0.0204 & 2.933 \\
\hline 0.05 & 10 & 0.5 & 0.1 & 0.1 & 0.7131 & 0.7306 & 0.2515 & -0.0875 & 3.7189 \\
\hline 0.05 & 8 & 0.5 & 0.5 & 0.5 & 0.5508 & 0.5562 & 0.3175 & 0.3224 & 3.4354 \\
\hline 0.05 & 8 & 0.5 & 0.6 & 0.6 & 0.5334 & 0.5354 & 0.3209 & 0.3535 & 3.3438 \\
\hline 3 & 5 & 1.5 & 0.5 & 0.5 & 1.5862 & 1.4800 & 0.5797 & 4.4762 & 167.305 \\
\hline 5 & 5 & 5 & 0.1 & 0.6 & 1.8104 & 1.6882 & 0.6583 & 4.5965 & 183.073 \\
\hline 5 & 5 & 5 & 0.5 & 0.5 & 1.7102 & 1.6066 & 0.6749 & 4.2527 & 177.0605 \\
\hline 5 & 5 & 5 & 0.9 & -0.9 & 1.8399 & 1.7654 & 0.7528 & 2.1844 & 26.3308 \\
\hline 0.1 & 15 & 1 & 0.9 & 0.9 & 0.6335 & 0.6611 & 0.279 & -0.198 & 2.3025 \\
\hline 0.1 & 15 & 0.5 & 0.9 & 0.9 & 0.7519 & 0.7787 & 0.2092 & 521.808 & 374033 \\
\hline
\end{tabular}

\subsection{Order Statistics and their moments}

Order statistics (OS) have wide applications in climatology, life testing and reliability. Moments of OS are also designed for replacement policy with the prediction of failure of future items determined from few early failures.

Let $X_{1}, \ldots, X_{n}$ be a random sample from the CRTMBIII model and let $X_{1: n}, \ldots, X_{n: n}$ be the corresponding order statistics. The pdf of the $\mathrm{i}^{\text {th }}$ order statistic, say $X_{i: n}$, is given by

$$
f_{i: n}(x)=\frac{f(x)}{B(i, n-i+1)} \sum_{r=0}^{n-i}(-1)^{r}\left(\begin{array}{c}
n-i \\
r
\end{array}\right) F^{r+i-1}(x),
$$

where $B(.,$.$) is the beta function.$

$$
\begin{aligned}
f_{i: n}(x)=\frac{1}{B(i, n-i+1)} \sum_{r=0}^{n-i} \sum_{s=0}^{r+i-1} \sum_{\ell=0}^{s} a_{r, s, \ell} \alpha \beta x^{-\beta-1}\left(1+\gamma x^{-\beta}\right)^{-\frac{\alpha}{\gamma}(s+r+2 \ell+i)-1} \\
\times\left[\lambda_{1}+2\left(\lambda_{2}-\lambda_{1}\right)\left(1+\gamma x^{-\beta}\right)^{-\frac{\alpha}{\gamma}}+3\left(1-\lambda_{2}\right)\left(1+\gamma x^{-\beta}\right)^{-\frac{2 \alpha}{\gamma}}\right],
\end{aligned}
$$


where $a_{r, s, \ell}=\lambda_{1}^{r+i-s-1}\left(\lambda_{2}-\lambda_{1}\right)^{s-\ell}\left(1-\lambda_{2}\right)^{\ell}(-1)^{r}\left(\begin{array}{c}n-i \\ r\end{array}\right)\left(\begin{array}{c}r+i-1 \\ s\end{array}\right)\left(\begin{array}{l}s \\ \ell\end{array}\right)$.

The $\mathrm{q}^{\text {th }}$ ordinary moment of $X_{i: n}$ say $\mu_{q}^{\prime}=E\left(X_{i: n}^{q}\right)$ is determined from (21) as

\subsection{Incomplete Moments}

Mean inactivity life; mean waiting time and inequality measures can be obtained from incomplete moments. For X CRTMBIII $\left(\alpha, \beta, \gamma, \lambda_{1}, \lambda_{2}\right)$, the incomplete lower moments are

$$
\begin{gathered}
M^{\prime}{ }_{r}(z)=E_{X \leq z}\left(X^{r}\right)=\int_{0}^{z} x^{r} \alpha \beta x^{-\beta-1}\left[\lambda_{1}\left(1+\gamma x^{-\beta}\right)^{-\frac{\alpha}{\gamma}-1}+2\left(\lambda_{2}-\lambda_{1}\right)(1+\right. \\
\left.\left.\gamma x^{-\beta}\right)^{-\frac{2 \alpha}{\gamma}-1}+3\left(1-\lambda_{2}\right)\left(1+\gamma x^{-\beta}\right)^{-\frac{3 \alpha}{\gamma}-1}\right] d x .
\end{gathered}
$$

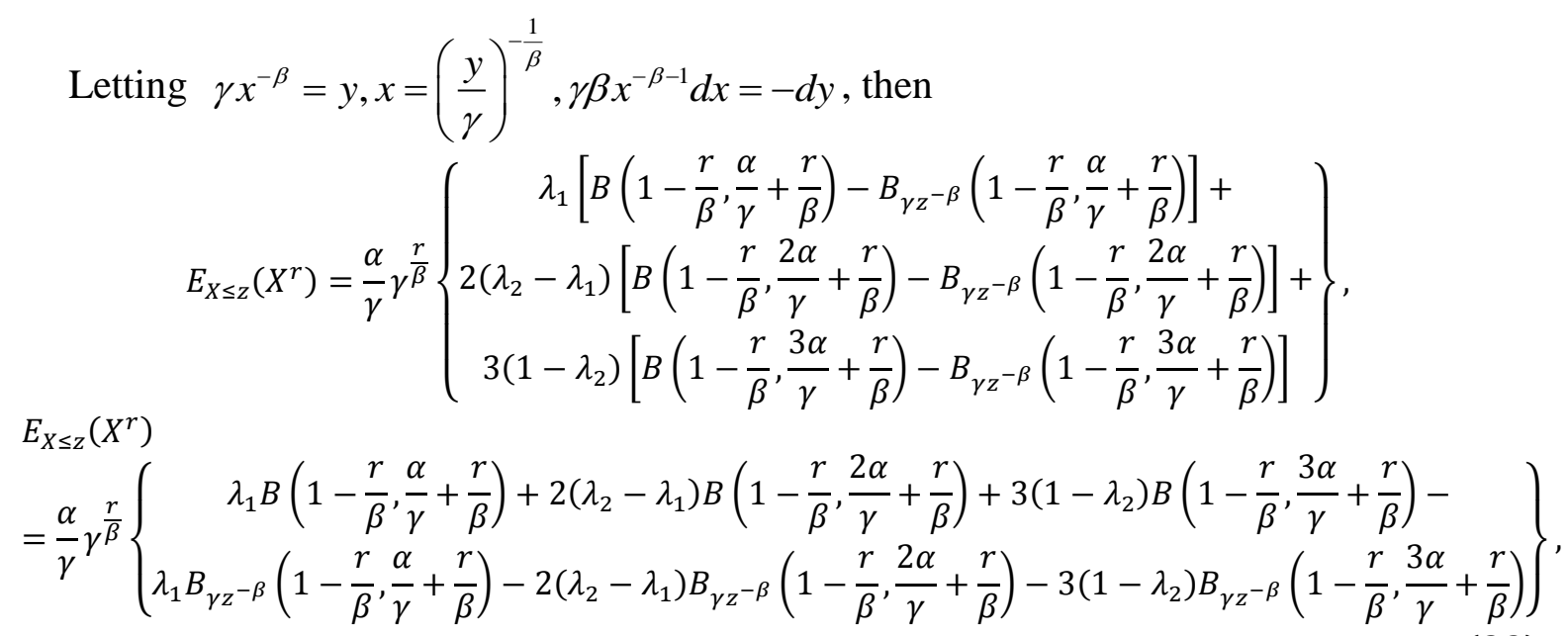

where $r<\beta$ and $B_{\gamma z^{-\beta}}(.,$.$) is the incomplete beta function.$

For X CRTMBIII $\left(\alpha, \beta, \gamma, \lambda_{1}, \lambda_{2}\right)$, the incomplete upper moments are

$$
\begin{aligned}
E_{X>Z}\left(X^{r}\right)=\int_{z}^{\infty} x^{r} & \alpha \beta x^{-\beta-1}\left[\lambda_{1}\left(1+\gamma x^{-\beta}\right)^{-\frac{\alpha}{\gamma}-1}+2\left(\lambda_{2}-\lambda_{1}\right)\left(1+\gamma x^{-\beta}\right)^{-\frac{2 \alpha}{\gamma}-1}\right. \\
& \left.+3\left(1-\lambda_{2}\right)\left(1+\gamma x^{-\beta}\right)^{-\frac{3 \alpha}{\gamma}-1}\right] d x
\end{aligned}
$$

Letting $\gamma x^{-\beta}=y, x=\left(\frac{y}{\gamma}\right)^{-\frac{1}{\beta}}, \gamma \beta x^{-\beta-1} d x=-d y$, we arrive at

$$
E_{X \geq Z}\left(X^{r}\right)=\frac{\alpha}{\gamma} \gamma^{\frac{r}{\beta}}\left[\begin{array}{r}
\lambda_{1} B_{\gamma z^{-\beta}}\left(1-\frac{r}{\beta}, \frac{\alpha}{\gamma}+\frac{r}{\beta}\right)+2\left(\lambda_{2}-\lambda_{1}\right) B_{\gamma z^{-\beta}}\left(1-\frac{r}{\beta}, \frac{2 \alpha}{\gamma}+\frac{r}{\beta}\right)+ \\
3\left(1-\lambda_{2}\right) B_{\gamma z^{-\beta}}\left(1-\frac{r}{\beta}, \frac{3 \alpha}{\gamma}+\frac{r}{\beta}\right)
\end{array}\right],
$$

where $r<\beta$ and $B_{\gamma z^{-\beta}}(.,$.$) is the incomplete beta function.$ 
The mean deviation about the mean $\left(\delta_{1}=E|X-\mu|\right)$ and about the median $\left(\delta_{2}=E|X-\tilde{\mu}|\right)$ can be written as $\delta_{1}=2 \mu F(\mu)-2 \mu M_{1}^{\prime}(\mu)$ and $\delta_{2}=\mu-2 M_{1}^{\prime}(\tilde{\mu})$ respectively, where $\mu=E(X)$ and $\tilde{\mu}=x_{0.5}$. The quantities $M_{1}^{\prime}(\mu)$ and $M_{1}^{\prime}(\tilde{\mu})$ can be obtained from (23). For specific probability p, Lorenz and Bonferroni curves are computed as $L(p)=\frac{M_{1}^{\prime}(q)}{\mu^{\prime}}, \mathrm{B}(p)=L(p) \mid p$ and, where $q=Q(p)$.

\subsection{Residual Life functions}

For X CRTMBIII $\left(\alpha, \beta, \gamma, \lambda_{1}, \lambda_{2}\right)$, the $\mathrm{n}^{\text {th }}$ moment of the residual life, $m_{n}(z)=$ $E\left[(X-z)^{n} \mid X>z\right]$, is

$$
\begin{gathered}
m_{n}(z)=\frac{1}{S(z)} \int_{z}^{\infty}(x-z)^{n} f(x) d x, \\
m_{n}(z)=\frac{1}{S(z)} \sum_{s=0}^{n}\left(\begin{array}{l}
n \\
s
\end{array}\right)(-z)^{n-s} E_{X>z}\left(X^{s}\right), \\
m_{n}(z)=\frac{1}{s(z)} \sum_{s=0}^{n}\left(\begin{array}{c}
n \\
s
\end{array}\right)(-z)^{n-s} \frac{\alpha}{\gamma} \gamma^{\frac{s}{\beta}}\left[\lambda_{1} B_{\gamma z^{-\beta}}\left(1-\frac{s}{\beta}, \frac{\alpha}{\gamma}+\frac{s}{\beta}\right)+2\left(\lambda_{2}-\right.\right. \\
\left.\left.\lambda_{1}\right) B_{\gamma z^{-\beta}}\left(1-\frac{s}{\beta}, \frac{2 \alpha}{\gamma}+\frac{s}{\beta}\right)+3\left(1-\lambda_{2}\right) B_{\gamma z^{-\beta}}\left(1-\frac{s}{\beta}, \frac{3 \alpha}{\gamma}+\frac{s}{\beta}\right)\right] .
\end{gathered}
$$

The average remaining lifetime of a component at time $\mathrm{z}$, say $m_{1}(z)$, or life expectancy is known as mean residual life (MRL) function is given by

$$
\begin{aligned}
m_{1}(z)=\frac{1}{S(z)} \sum_{s=0}^{1}\left(\begin{array}{l}
1 \\
s
\end{array}\right)(-z)^{1-s} \frac{\alpha}{\gamma} \gamma^{\frac{s}{\beta}}\left[\lambda_{1} B_{\gamma z^{-\beta}}\left(1-\frac{s}{\beta}, \frac{\alpha}{\gamma}+\frac{s}{\beta}\right)\right. \\
\left.+2\left(\lambda_{2}-\lambda_{1}\right) B_{\gamma z^{-\beta}}\left(1-\frac{s}{\beta}, \frac{2 \alpha}{\gamma}+\frac{s}{\beta}\right)+3\left(1-\lambda_{2}\right) B_{\gamma z^{-\beta}}\left(1-\frac{s}{\beta}, \frac{3 \alpha}{\gamma}+\frac{s}{\beta}\right)\right] .
\end{aligned}
$$

For $\mathrm{X} 1 \sim \operatorname{CRTMBIII}\left(\alpha, \beta, \gamma, \lambda_{1}, \lambda_{2}\right)$, the nth moment of the reverse residual life, $M_{n}(z)=$ $E\left[(z-X)^{n} \mid X \leq z\right]$ is

$$
\begin{aligned}
& M_{n}(z)=\frac{1}{F(z)} \int_{0}^{z}(z-x)^{n} f(x) d x \\
& M_{n}(z)=\frac{1}{F(z)} \sum_{s=0}^{n}(-1)^{s}\left(\begin{array}{l}
n \\
s
\end{array}\right) z^{n-s} E_{X \leq z}\left(X^{s}\right) \text {, }
\end{aligned}
$$

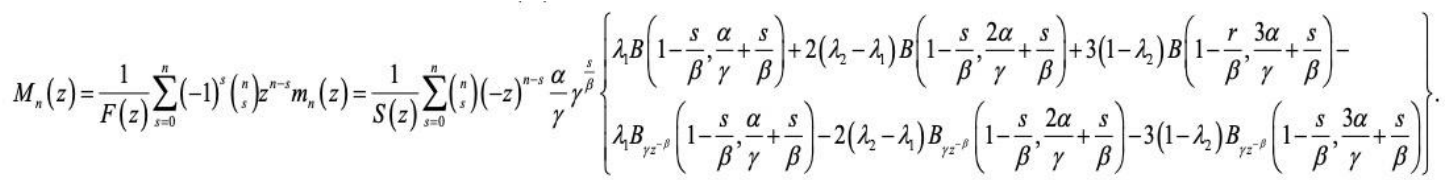

The waiting time $\mathrm{z}$ for failure of a component has passed with condition that this failure had happened in the interval $[0, \mathrm{z}]$ is called mean waiting time (MWT) or mean inactivity 
time. The waiting time $\mathrm{z}$ for failure of a component of $\mathrm{X}$ with CRTMBIII distribution is defined by

$$
M_{1}(z)=\frac{1}{F(z)} \sum_{s=0}^{1}(-1) s\left(\begin{array}{l}
1 \\
s
\end{array}\right) z^{1-s} \frac{\alpha}{\gamma} \gamma^{\frac{s}{\beta}}\left\{\begin{array}{l}
\lambda_{1} B\left(1-\frac{s}{\beta}, \frac{\alpha}{\gamma}+\frac{s}{\beta}\right)+2\left(\lambda_{2}-\lambda_{1}\right) B\left(1-\frac{s}{\beta}, \frac{2 \alpha}{\gamma}+\frac{s}{\beta}\right)+3\left(1-\lambda_{2}\right) B\left(1-\frac{r}{\beta}, \frac{3 \alpha}{\gamma}+\frac{s}{\beta}\right)- \\
\lambda_{1} B_{\gamma z^{-\beta}}\left(1-\frac{s}{\beta}, \frac{\alpha}{\gamma}+\frac{s}{\beta}\right)-2\left(\lambda_{2}-\lambda_{1}\right) B_{\gamma z^{-\beta}}\left(1-\frac{s}{\beta}, \frac{2 \alpha}{\gamma}+\frac{s}{\beta}\right)-3\left(1-\lambda_{2}\right) B_{\gamma z^{-\beta}}\left(1-\frac{s}{\beta}, \frac{3 \alpha}{\gamma}+\frac{s}{\beta}\right)
\end{array}\right\}
$$

\subsection{Stress-strength Reliability for CRTMBIII Distribution}

Let $\mathrm{X}_{1} \sim \operatorname{CRTMBII}\left(\alpha_{1}, \beta, \gamma, \lambda_{1}, \lambda_{2}\right)$ and $\mathrm{X}_{2} \sim \operatorname{CRTMBII}\left(\alpha_{2}, \beta, \gamma, \lambda_{1}, \lambda_{2}\right)$, such that $X_{1}$ represents strength and $X_{2}$ represents stress. Then reliability of the component is:

$$
\begin{aligned}
& \mathrm{R}=\operatorname{Pr}\left(\mathrm{X}_{2}<X_{1}\right)=\int_{-\infty}^{\infty} \int_{-\infty}^{x_{1}} f\left(x_{1}, x_{2}\right) d x_{2} d x_{1}=\int_{0}^{\infty} f_{x_{1}}(x) F_{x_{2}}(x) d x \\
& R=\int_{0}^{\infty} \alpha_{1} \beta x^{-\beta-1}\left[\lambda_{1}\left(1+\gamma x^{-\beta}\right)^{-\frac{\alpha_{1}}{\gamma}-1}+2\left(\lambda_{2}-\lambda_{1}\right)\left(1+\gamma x^{-\beta}\right)^{-\frac{2 \alpha_{1}}{\gamma}-1}+3\left(1-\lambda_{2}\right)\left(1+\gamma x^{-\beta}\right)^{-\frac{3 \alpha_{1}}{\gamma}-1}\right] \times \\
& {\left[\lambda_{1}\left(1+\gamma x^{-\beta}\right)^{-\frac{\alpha_{2}}{\gamma}}+\left(\lambda_{2}-\lambda_{1}\right)\left(1+\gamma x^{-\beta}\right)^{-\frac{2 \alpha_{2}}{\gamma}}+\left(1-\lambda_{2}\right)\left(1+\gamma x^{-\beta}\right)^{-\frac{3 \alpha_{2}}{\gamma}}\right] d x,} \\
& R=\left\{\begin{array}{l}
\int_{0}^{\infty} \alpha_{1} \beta x^{-\beta-1}\left[\lambda_{1}^{2}\left(1+\gamma x^{-\beta}\right)^{-\frac{\alpha_{1}}{\gamma}-\frac{\alpha_{2}}{\gamma}-1}+2 \lambda_{1}\left(\lambda_{2}-\lambda_{1}\right)\left(1+\gamma x^{-\beta}\right)^{-\frac{2 \alpha_{1}}{\gamma}-\frac{\alpha_{2}}{\gamma}-1}+3\left(\lambda_{1}-\lambda_{1} \lambda_{2}\right)\left(1+\gamma x^{-\beta}\right)^{-\frac{3 \alpha_{1}}{\gamma}-\frac{\alpha_{2}}{\gamma}-1}\right]+ \\
\int_{0}^{\infty} \alpha_{1} \beta x^{-\beta-1}\left[\left(\lambda_{1} \lambda_{2}-\lambda_{1}^{2}\right)\left(1+\gamma x^{-\beta}\right)^{-\frac{\alpha_{1}}{\gamma}-\frac{2 \alpha_{2}}{\gamma}-1}+2\left(\lambda_{2}-\lambda_{1}\right)^{2}\left(1+\gamma x^{-\beta}\right)^{-\frac{2 \alpha_{1}}{\gamma}-\frac{2 \alpha_{2}}{\gamma}-1}+3\left(1-\lambda_{2}\right)\left(\lambda_{2}-\lambda_{1}\right)\left(1+\gamma x^{-\beta}\right)^{-\frac{3 \alpha_{1}}{\gamma}-\frac{2 \alpha_{2}}{\gamma}-1}\right]+ \\
\int_{0}^{\infty} \alpha_{1} \beta x^{-\beta-1}\left[\left(\lambda_{1}-\lambda_{1} \lambda_{2}\right)\left(1+\gamma x^{-\beta}\right)^{-\frac{\alpha_{1}}{\gamma}-\frac{3 \alpha_{2}}{\gamma}-1}+2\left(\lambda_{2}-\lambda_{1}\right)\left(1-\lambda_{2}\right)\left(1+\gamma x^{-\beta}\right)^{-\frac{2 \alpha_{1}}{\gamma}-\frac{3 \alpha_{2}}{\gamma}-1}+3\left(1-\lambda_{2}\right)^{2}\left(1+\gamma x^{-\beta}\right)^{-\frac{3 \alpha_{1}}{\gamma}-\frac{3 \alpha_{2}}{\gamma}-1}\right]
\end{array}\right\}, \\
& R=\left\{\begin{array}{l}
\frac{\lambda_{1}^{2} \alpha_{1}}{\left(\alpha_{1}+\alpha_{2}\right)}+\frac{2 \alpha_{1}\left(\lambda_{1} \lambda_{2}-\lambda_{1}^{2}\right)}{\left(2 \alpha_{1}+\alpha_{2}\right)}+\frac{3 \alpha_{1}\left(\lambda_{1}-\lambda_{1} \lambda_{2}\right)}{\left(3 \alpha_{1}+\alpha_{2}\right)}+ \\
\frac{\alpha_{1}\left(\lambda_{2}-\lambda_{1}\right)^{2}}{\left(\alpha_{1}+2 \alpha_{2}\right)}+\frac{2 \alpha_{1}\left(\lambda_{1} \lambda_{2}-\lambda_{1}^{2}\right)}{\left(2 \alpha_{1}+2 \alpha_{2}\right)}+\frac{3 \alpha_{1}\left(1-\lambda_{2}\right)\left(\lambda_{2}-\lambda_{1}\right)}{\left(3 \alpha_{1}+2 \alpha_{2}\right)}+ \\
\frac{\alpha_{1}\left(\lambda_{1}-\lambda_{1} \lambda_{2}\right)}{\left(\alpha_{1}+3 \alpha_{2}\right)}+\frac{2 \alpha_{1}\left(\lambda_{2}-\lambda_{1}\right)\left(1-\lambda_{2}\right)}{\left(2 \alpha_{1}+3 \alpha_{2}\right)}+\frac{3 \alpha_{1}\left(1-\lambda_{2}\right)^{2}}{\left(3 \alpha_{1}+3 \alpha_{2}\right)}
\end{array}\right\} .
\end{aligned}
$$

Therefore the stress-strength reliability parameter $\mathrm{R}$ is independent of parameters $\beta, \gamma, \lambda_{1}$ and $\lambda_{2}$.

\section{CHARACTERIZATIONS}

In order to develop a stochastic function in a certain problem, it is necessary to know whether the selected function fulfills the requirements of the specific underlying probability distribution. To this end, it is required to study characterizations of the specific probability distribution. Here, we present two characterizations of the CRTMBIII distribution (i) ratio of the truncated moments and (ii) double truncated moments.

\subsection{Ratio of Truncated Moments}

We characterize the CRTMBIII distribution on the basis of a simple relationship between two truncated moments of functions of X [Theorem G (Glänzel; 1987)]. 
Proportion 4.1.1: Let $X: \Omega \rightarrow(0, \infty)$ be a continuous random variable and let

$$
h_{1}(x)=\frac{1}{\alpha}\left[\lambda_{1}\left(1+\gamma x^{-\beta}\right)^{-\frac{\alpha}{\gamma}-1}+2\left(\lambda_{2}-\lambda_{1}\right)\left(1+\gamma x^{-\beta}\right)^{-\frac{2 \alpha}{\gamma}-1}+3\left(1-\lambda_{2}\right)\left(1+\gamma x^{-\beta}\right)^{-\frac{3 \alpha}{\gamma}-1}\right]^{-1}
$$

and

$$
h_{2}(x)=\frac{2}{\alpha} x^{-\beta}\left[\lambda_{1}\left(1+\gamma x^{-\beta}\right)^{-\frac{\alpha}{\gamma}-1}+2\left(\lambda_{2}-\lambda_{1}\right)\left(1+\gamma x^{-\beta}\right)^{-\frac{2 \alpha}{\gamma}-1}+3\left(1-\lambda_{2}\right)\left(1+\gamma x^{-\beta}\right)^{-\frac{3 \alpha}{\gamma}-1}\right]^{-1}, x>0 .
$$

The pdf of $\mathrm{X}$ is (10), if and only if $q(x)$ (in Theorem $\mathrm{G}$ ) has the form $q(x)=x^{\beta}, x>0$.

Proof. If $X$ has pdf (10), then

$$
\begin{gathered}
(1-F(x)) E\left(h_{1}(x) \mid X \geq x\right)=x^{-\beta}, x>0, \\
(1-F(x)) E\left(h_{2}(x) \mid X \geq x\right)=x^{-2 \beta}, x>0,
\end{gathered}
$$

and

$$
\frac{E\left[\mathrm{~h}_{1}(X) \mid \mathrm{X} \geq \mathrm{x}\right]}{E\left[\mathrm{~h}_{2}(X) \mid \mathrm{X} \geq \mathrm{x}\right]}=q(x)=x^{\beta}
$$

The differential equation $s^{\prime}(x)=\frac{q^{\prime}(x) h_{2}(x)}{q(x) h_{2}(x)-h_{1}(x)}=\frac{2 \beta}{x}$ has solution $(x)=\ln x^{2 \beta}$.

Therefore according to theorem $\mathrm{G}, \mathrm{X}$ has pdf (11).

Corollary 4.1.1: Let $X: \Omega \rightarrow(0, \infty)$ be a continuous random variable and let

$$
h_{2}(x)=\frac{2}{\alpha} x^{-\beta}\left[\lambda_{1}\left(1+\gamma x^{-\beta}\right)^{-\frac{\alpha}{\gamma}-1}+2\left(\lambda_{2}-\lambda_{1}\right)\left(1+\gamma x^{-\beta}\right)^{-\frac{2 \alpha}{\gamma}-1}+3\left(1-\lambda_{2}\right)\left(1+\gamma x^{-\beta}\right)^{-\frac{3 \alpha}{\gamma}-1}\right]^{-1}, x>0 .
$$

The pdf of $\mathrm{X}$ is (10) if and only if functions $q(x)$ and $h_{1}(x)$ satisfy the equation

$$
\frac{q^{\prime}(x) h_{2}(x)}{q(x) h_{2}(x)-h_{1}(x)}=\frac{2 \beta}{x} \text {. }
$$

Remark 4.1.1: The general solution of the above differential equation is

$$
q(x)=x^{2 \beta}\left[-\int 2 \beta x^{-(2 \beta+1)}\left(h_{2}(x)\right)^{-1} h_{1}(x) d x+D\right],
$$

where $\mathrm{D}$ is a constant.

\subsection{Doubly Truncated Moment}

Here, we characterize the CRTMBIII distribution via doubly truncated moment.

Proposition 4.2.1: Let $\mathrm{X}: \Omega \rightarrow(0,+\infty)$ be a continuous random variable. Then $\mathrm{X}$ has pdf (10) if and only if

$$
\begin{aligned}
E\left[\left[\lambda_{1}+2\left(\lambda_{2}-\lambda_{1}\right)\left(1+\gamma x^{-\beta}\right)^{-\frac{\alpha}{\gamma}}\right.\right. & \left.\left.+3\left(1-\lambda_{2}\right)\left(1+\gamma x^{-\beta}\right)^{-\frac{2 \alpha}{\gamma}}\right]^{-1} \mid x<\mathrm{X}<y\right] \\
& =\frac{\left(1+\gamma y^{-\beta}\right)^{-\frac{\alpha}{\gamma}}-\left(1+\gamma x^{-\beta}\right)^{-\frac{\alpha}{\gamma}}}{[\bar{F}(x)-\bar{F}(y)]}, x>0, y>0 .
\end{aligned}
$$

\section{Proof:}

For random variable $\mathrm{X}$ with pdf (10), we have 


$$
\begin{gathered}
E\left[\left[\lambda_{1}+2\left(\lambda_{2}-\lambda_{1}\right)\left(1+\gamma x^{-\beta}\right)^{-\frac{\alpha}{\gamma}}+3\left(1-\lambda_{2}\right)\left(1+\gamma x^{-\beta}\right)^{-\frac{2 \alpha}{\gamma}}\right]^{-1} \mid x<\mathrm{X}<y\right] \\
=\frac{\int_{y}^{x}\left[\lambda_{1}+2\left(\lambda_{2}-\lambda_{1}\right)\left(1+\gamma u^{-\beta}\right)^{-\frac{\alpha}{\gamma}}+3\left(1-\lambda_{2}\right)\left(1+\gamma u^{-\beta}\right)^{-\frac{2 \alpha}{\gamma}}\right]^{-1} f(u) d u}{[\bar{F}(x)-\bar{F}(y)]}, \\
=\frac{\int_{y}^{x}\left[\lambda_{1}+2\left(\lambda_{2}-\lambda_{1}\right)\left(1+\gamma u^{-\beta}\right)^{-\frac{\alpha}{\gamma}}+3\left(1-\lambda_{2}\right)\left(1+\gamma u^{-\beta}\right)^{-\frac{2 \alpha}{\gamma}}\right]^{-1} \alpha \beta u^{-\beta-1}\left(1+\gamma u^{-\beta}\right)^{-\frac{\alpha}{\gamma}}\left[\lambda_{1}+2\left(\lambda_{2}-\lambda_{1}\right)\left(1+\gamma u^{-\beta}\right)^{-\frac{\alpha}{\gamma}}+3\left(1-\lambda_{2}\right)\left(1+\gamma u^{-\beta}\right)^{-\frac{2 \alpha}{\gamma}}\right] d u}{[\bar{F}(x)-\bar{F}(y)]}, \\
=\frac{\int_{y}^{x} \alpha \beta u^{-\beta-1}\left(1+\gamma u^{-\beta}\right)^{-\frac{\alpha}{\gamma}-1} d u}{[F(y)-F(x)]}=\frac{\left(1+\gamma y^{-\beta}\right)^{-\frac{\alpha}{\gamma}}-\left(1+\gamma x^{-\beta}\right)^{-\frac{\alpha}{\gamma}}}{[\bar{F}(x)-\bar{F}(y)]} .
\end{gathered}
$$

Conversely, if (30) holds, then

$$
\begin{aligned}
& \frac{\int_{y}^{x}\left[\lambda_{1}+2\left(\lambda_{2}-\lambda_{1}\right)\left(1+\gamma u^{-\beta}\right)^{-\frac{\alpha}{\gamma}}+3\left(1-\lambda_{2}\right)\left(1+\gamma u^{-\beta}\right)^{-\frac{2 \alpha}{\gamma}}\right]^{-1} f(u) d u}{[\bar{F}(x)-\bar{F}(y)]}=\frac{\left(1+\gamma y^{-\beta}\right)^{-\frac{\alpha}{\gamma}}-\left(1+\gamma x^{-\beta}\right)^{-\frac{\alpha}{\gamma}}}{[\bar{F}(x)-\bar{F}(y)]} \\
& \int_{y}^{x}\left[\lambda_{1}+2\left(\lambda_{2}-\lambda_{1}\right)\left(1+\gamma u^{-\beta}\right)^{-\frac{\alpha}{\gamma}}+3\left(1-\lambda_{2}\right)\left(1+\gamma u^{-\beta}\right)^{-\frac{2 \alpha}{\gamma}}\right]^{-1} f(u) d u=\left(1+\gamma y^{-\beta}\right)^{-\frac{\alpha}{\gamma}}-\left(1+\gamma x^{-\beta}\right)^{-\frac{\alpha}{\gamma}} .
\end{aligned}
$$

Differentiating with respect to y, we have

$$
\left[\lambda_{1}+2\left(\lambda_{2}-\lambda_{1}\right)\left(1+\gamma y^{-\beta}\right)^{-\frac{\alpha}{\gamma}}+3\left(1-\lambda_{2}\right)\left(1+\gamma y^{-\beta}\right)^{-\frac{2 \alpha}{\gamma}}\right]^{-1} f(y)=\alpha \beta y^{-\beta-1}\left(1+\gamma y^{-\beta}\right)^{-\frac{\alpha}{\gamma}-1}
$$

or

$$
f(x)=\alpha \beta x^{-\beta-1}\left(1+\gamma x^{-\beta}\right)^{-\frac{\alpha}{\gamma}-1}\left[\lambda_{1}+2\left(\lambda_{2}-\lambda_{1}\right)\left(1+\gamma x^{-\beta}\right)^{-\frac{\alpha}{\gamma}}+3\left(1-\lambda_{2}\right)\left(1+\gamma x^{-\beta}\right)^{-\frac{2 \alpha}{\gamma}}\right], x>0,
$$

which is pdf of the CRTMBIII distribution.

\section{MAXIMUM LIKELIHOOD ESTIMATION}

Here, we adopt maximum likelihood estimation technique for the CRTMBIII parameters. Let $\Phi=\left(\alpha, \beta, \gamma, \lambda_{1}, \lambda_{2}\right)$ be unknown parameter vector. The log likelihood function $\ell(\Phi)$ for the CRTMBIII distribution is

We can compute the maximum likelihood estimators (MLEs) of $\alpha, \beta, \gamma, \lambda_{1}, \lambda_{2}$ by solving equations 32-36 either directly or using quasi-Newton procedure, computer packages/ softwares such as R, SAS, Ox, MATLAB, MAPLE and MATHEMATICA,. 


$$
\begin{aligned}
& \frac{\partial}{\partial \alpha}\left(\operatorname{lnL}\left(x_{i} ; \Phi\right)\right)=\frac{n}{\alpha}-\frac{1}{\gamma} \sum \ln \left(1+\gamma x_{i}^{-\beta}\right)-\frac{2}{\gamma} \sum \frac{\left(1+\gamma x_{i}^{-\beta}\right)^{-\frac{\alpha}{\gamma}} \ln \left(1+\gamma x_{i}^{-\beta}\right)\left[\left(\lambda_{2}-\lambda_{1}\right)+3\left(1-\lambda_{2}\right)\left(1+\gamma x_{i}^{-\beta}\right)^{-\frac{\alpha}{\gamma}}\right]}{\left[\lambda_{1}+2\left(\lambda_{2}-\lambda_{1}\right)\left(1+\gamma x_{i}^{-\beta}\right)^{-\frac{\alpha}{\gamma}}+3\left(1-\lambda_{2}\right)\left(1+\gamma x_{i}^{-\beta}\right)^{-\frac{2 \alpha}{\gamma}}\right]}=0, \\
& \frac{\partial}{\partial \beta}\left(\ln L\left(x_{i} ; \Phi\right)\right)=\frac{n}{\beta}-\sum \ln x_{i}+\left(\frac{\alpha}{\gamma}+1\right) \sum \frac{\gamma x_{i}^{-\beta} \ln x_{i}}{\left(1+\gamma x_{i}^{-\beta}\right)}+2 \sum x_{i}^{-\beta} \ln x_{i}\left(1+\gamma x_{i}^{-\beta}\right)^{-\frac{\alpha}{\gamma}-1}\left\{\frac{\alpha\left(\lambda_{2}-\lambda_{1}\right)+3 \alpha\left(1-\lambda_{2}\right)\left(1+\gamma x_{i}^{-\beta}\right)^{-\frac{\alpha}{\gamma}}}{\lambda_{1}+2\left(\lambda_{2}-\lambda_{1}\right)\left(1+\gamma x_{i}^{-\beta}\right)^{-\frac{\alpha}{\gamma}}+3\left(1-\lambda_{2}\right)\left(1+\gamma x_{i}^{-\beta}\right)^{-\frac{2 \alpha}{\gamma}}}\right\}=0(33) \\
& \frac{\partial}{\partial \gamma}\left(\ln L\left(x_{i}, \Phi\right)\right)=\frac{\alpha}{\gamma^{2}} \sum \ln \left(1+\gamma x_{i}^{-\beta}\right)-\left(1+\frac{\alpha}{\gamma}\right) \sum x_{i}^{-\beta}\left(1+\gamma x_{i}^{-\beta}\right)^{-1}+\frac{2 \alpha}{\gamma} \sum \frac{\left\{\frac{\left(\lambda_{2}-\lambda_{1}\right)}{\left(1+\gamma x_{i}^{-\beta}\right)^{\frac{\alpha}{\gamma}}}\left[\frac{1}{\gamma} \ln \left(1+\gamma x_{i}^{-\beta}\right)-\frac{x_{i}^{-\beta}}{\left(1+\gamma x_{i}^{-\beta}\right)}\right]+\frac{3\left(1-\lambda_{2}\right)}{\left(1+\gamma x_{i}^{-\beta}\right)^{-\frac{2 \alpha}{\gamma}}}\left[\frac{\alpha}{\gamma^{2}} \ln \left(1+\gamma x_{i}^{-\beta}\right)-\frac{x_{i}^{-\beta}}{\left(1+\gamma x_{i}^{-\beta}\right)}\right]\right\}}{\left[\lambda_{1}+2\left(\lambda_{2}-\lambda_{1}\right)\left(1+\gamma x_{i}^{-\beta}\right)^{-\frac{\alpha}{\gamma}}+3\left(1-\lambda_{2}\right)\left(1+\gamma x_{i}^{-\beta}\right)^{-\frac{2 \alpha}{\gamma}}\right]}=0,(34) \\
& \frac{\partial}{\partial \lambda_{1}}\left(\operatorname{lnL}\left(x_{i} ; \Phi\right)\right)=\sum\left[1-2\left(1+\gamma x_{i}^{-\beta}\right)^{-\frac{\alpha}{\gamma}}\right]\left[\lambda_{1}+2\left(\lambda_{2}-\lambda_{1}\right)\left(1+\gamma x_{i}^{-\beta}\right)^{-\frac{\alpha}{\gamma}}+3\left(1-\lambda_{2}\right)\left(1+\gamma x_{i}^{-\beta}\right)^{-\frac{2 \alpha}{\gamma}}\right]^{-1}=0 \text {, } \\
& \frac{\partial}{\partial \lambda_{2}}\left(\operatorname{lnL}\left(x_{i} ; \Phi\right)\right)=\sum\left[2\left(1+\gamma x_{i}^{-\beta}\right)^{-\frac{\alpha}{\gamma}}-3\left(1+\gamma x_{i}^{-\beta}\right)^{-\frac{2 \alpha}{\gamma}}\right]\left[\lambda_{1}+2\left(\lambda_{2}-\lambda_{1}\right)\left(1+\gamma x_{i}^{-\beta}\right)^{-\frac{\alpha}{\gamma}}+3\left(1-\lambda_{2}\right)\left(1+\gamma x_{i}^{-\beta}\right)^{-\frac{2 \alpha}{\gamma}}\right]^{-1}=0 \text {. }
\end{aligned}
$$

\section{SIMULATION STUDY}

In this section, we survey the performance of the MLEs of the parameters of the CRTMBIII distribution with respect to sample size $n$. This performance is done based on the following simulation study:

Step 1: Generate 1000 samples of size $n$ from the CRTMBIII distribution based on the inverse cdf method.

Step 2: Compute the MLEs for 1000 samples, say $\left(\alpha, \beta, \gamma, \lambda_{1}, \lambda_{2}\right)$ for $i=1,2, \ldots, 1000$ based on non-linear optimization algorithm with constraint matching to range of parameters.

Step 3: Compute the biases, mean squared errors and coverage probability of MLEs.

For this purpose, we have selected different arbitrarily parameter values and $n=50,100,150,200$ sample sizes. All codes are written in $\mathrm{R}$ and the results are summarized in Table 3. The result clearly shows that when the sample size increases, the mean square errors (MSEs) decrease. This shows the consistency of MLE estimators.

Table 3: Bias, MSEs and coverage probability (CP) of the CRTMBIII $(1,8,1,0.5,0.5)$, $(2,3,1,0.5,0.5)(0.5,5,1.2,0.9,0.9)$ distribution.

\begin{tabular}{lcccccc}
\hline \hline Sample & Statistics & $\alpha=1$ & $\beta=8$ & $\gamma=1$ & $\lambda_{1}=0.5$ & $\lambda_{2}=0.5$ \\
& & & & & & \\
\hline \hline \multirow{2}{*}{$\mathbf{n} \mathbf{5 0}$} & Bias & 0.472 & 1.638 & 0.761 & 0.104 & 0.180 \\
& MSE & 0.992 & 3.507 & 1.329 & 0.867 & 0.772 \\
& CP & 94.90 & 95.12 & 94.11 & 95.23 & 94.97 \\
\hline \multirow{2}{*}{$\mathbf{n = 1 0 0}$} & Bias & 0.289 & 1.419 & 0.434 & 0.091 & 0.094 \\
& MSE & 0.718 & 1.127 & 1.071 & 0.530 & 0.423 \\
& CP & 95.30 & 94.89 & 95.04 & 95.01 & 94.99 \\
\hline \multirow{2}{*}{$\mathbf{2 0 0}$} & Bias & 0.117 & 0.912 & 0.210 & 0.070 & 0.087 \\
& MSE & 0.482 & 1.067 & 0.975 & 0.313 & 0.189 \\
& CP & 95.00 & 94.98 & 94.99 & 94.95 & 95.04 \\
\hline \multirow{2}{*}{$\mathbf{n}=\mathbf{3 0 0}$} & Bias & 0.073 & 0.150 & 0.097 & 0.003 & 0.050 \\
& MSE & 0.210 & 0.829 & 0.151 & 0.113 & 0.093 \\
& CP & 95.07 & 95.01 & 94.95 & 94.99 & 95.02 \\
\hline
\end{tabular}




\begin{tabular}{|c|c|c|c|c|c|c|}
\hline Sample & Statistics & $\alpha=3$ & $\beta=2$ & $\gamma=1$ & $\lambda_{1}=0.5$ & $\lambda_{2}=0.5$ \\
\hline \multirow{3}{*}{$\mathbf{n}=\mathbf{5 0}$} & Bias & 0.846 & 0.721 & 0.779 & 0.112 & 0.177 \\
\hline & MSE & 1.232 & 1.221 & 1.211 & 0.921 & 0.646 \\
\hline & $\mathrm{CP}$ & 95.10 & 94.97 & 94.64 & 95.09 & 94.98 \\
\hline \multirow{3}{*}{$n=100$} & Bias & 0.789 & 0.544 & 0.452 & 0.095 & 0.820 \\
\hline & MSE & 0.921 & 0.988 & 1.010 & 0.621 & 0.524 \\
\hline & $\mathrm{CP}$ & 95.03 & 95.09 & 94.93 & 94.96 & 95.04 \\
\hline \multirow{3}{*}{$n=200$} & Bias & 0.327 & 0.477 & 0.188 & 0.087 & 0.066 \\
\hline & MSE & 0.768 & 0.661 & 0.923 & 0.320 & 0.168 \\
\hline & $\mathrm{CP}$ & 95.09 & 95.04 & 95.03 & 95.05 & 95.02 \\
\hline \multirow{3}{*}{$n=300$} & Bias & 0.112 & 0.187 & 0.078 & 0.005 & 0.032 \\
\hline & MSE & 0.395 & 0.522 & 0.130 & 0.121 & 0.104 \\
\hline & $\mathrm{CP}$ & 94.99 & 95.03 & 95.01 & 95.03 & 94.99 \\
\hline \multirow[t]{2}{*}{ Sample } & Statistics & $\alpha=0.5$ & $\beta=5$ & $\gamma=1.2$ & $\lambda=0.9$ & $\lambda_{2}=0.9$ \\
\hline & Bias & 0.112 & 1.822 & 0.323 & 0.298 & 0.310 \\
\hline \multirow[t]{2}{*}{$\mathbf{n}=\mathbf{5 0}$} & MSE & 0.315 & 2.301 & 0.748 & 0.664 & 0.734 \\
\hline & $\mathrm{CP}$ & 95.08 & 95.61 & 96.06 & 94.78 & 95.22 \\
\hline \multirow{3}{*}{$n=100$} & Bias & 0.099 & 0.921 & 0.214 & 0.166 & 0.201 \\
\hline & MSE & 0.277 & 1.321 & 0.432 & 0.422 & 0.512 \\
\hline & $\mathrm{CP}$ & 95.10 & 94.93 & 95.08 & 94.97 & 95.09 \\
\hline \multirow{3}{*}{$\mathbf{n}=\mathbf{2 0 0}$} & Bias & 0.046 & 0.733 & 0.181 & 0.084 & 0.092 \\
\hline & MSE & 0.121 & 0.887 & 0.312 & 0.128 & 0.196 \\
\hline & $\mathrm{CP}$ & 95.04 & 95.31 & 95.09 & 95.05 & 94.89 \\
\hline \multirow{3}{*}{$n=300$} & Bias & 0.010 & 0.171 & 0.084 & 0.055 & 0.069 \\
\hline & MSE & 0.111 & 0.634 & 0.139 & 0.105 & 0.113 \\
\hline & $\mathrm{CP}$ & 95.03 & 94.98 & 95.12 & 95.00 & 95.06 \\
\hline
\end{tabular}

\section{APPLICATIONS}

We consider an application to data set such as strengths of $1.5 \mathrm{~cm}$ glass fibers for authentication of the flexibility, utility and potentiality of the CRTMBIII distribution. We compare the CRTMBIII distribution with TMBIII, MBIII, BIII, LL distributions. For selection of the optimum distribution, we compute the estimate of likelihood ratio statistics $(-2 \hat{\ell})$, Akaike information criterion (AIC), corrected Akaike information criterion (CAIC), Bayesian information criterion (BIC), Hannan-Quinn information criterion (HQIC), Cramervon Mises ( $\left.\mathrm{W}^{*}\right)$, Anderson Darling (A*), and Kolmogorov- Smirnov [K-S] statistics with pvalues for all competing and sub distributions. We compute the MLEs and their standard errors (in parentheses). We also compute goodness of fit statistics (GOFs) values for the CRTMBIII, TMBIII, MBIII, BIII, LL models.

7.1 Strengths of Glass Fibers: The values of data about strengths of $1.5 \mathrm{~cm}$ glass fibers (Smith and Naylor; 1987 and Arifa et al.;2017) are: 0.55, 0.74, 0.77, 0.81, 0.84, 0.93, 1.04, $1.11,1.13,1.24,1.25,1.27,1.28,1.29,1.30,1.36,1.39,1.42,1.48,1.48,1.49,1.49,1.50$, $1.50,1.51,1.52,1.53,1.54,1.55,1.55,1.58,1.59,1.60,1.61,1.61,1.61,1.61,1.62,1.62$, $1.63,1.64,1.66,1.66,1.66,1.67,1.68,1.68,1.69,1.70,1.70,1.73,1.76,1.76,1.77,1.78$, $1.81,1.82,1.84,1.84,1.89,2.00,2.01,2.24$.

A descriptive summary for the strengths of $1.5 \mathrm{~cm}$ glass fibers data set provides the following values: 63 (sample size), 0.55 (minimum), 2.24 (maximum), 1.59 (median), 1.506825 (mean), 0.3241257 (standard deviation), 21.5105 (coefficient of variation), 0.89993 (coefficient of skewness) and 3.92376 (coefficient of kurtosis). The boxplot (Fig. 
3(a)) for strengths of glass fibers data is negatively skewed. The TTT (total time on test) plot (Fig. 3(b)) for strengths of glass fibers data is concave, which infers increasing failure rate. So, the BIII-ME distribution is suitable to model these data.

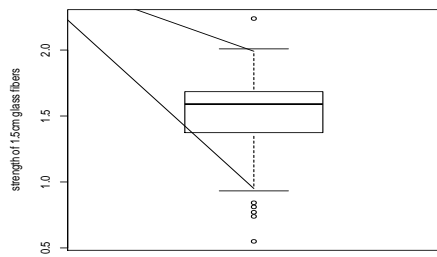

(a)

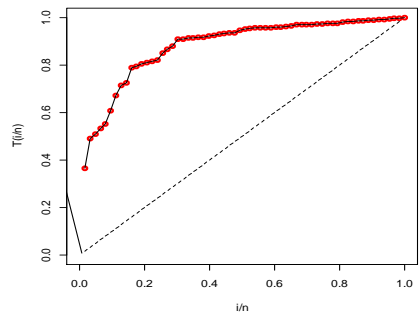

(b)

Figure 3 Boxplot (a) and TTT plot (b) for glass fiber data

Table 4 reports the MLEs (standard errors) and measures $\mathrm{W}^{*}, \mathrm{~A}^{*}, \mathrm{KS}$ (p-values). Table 5 displays the values $-2 \hat{\ell}$, AIC, CAIC, BIC and HQIC.

Table 4: MLEs, (standard errors) and $\mathrm{W}^{*}, \mathrm{~A}^{*}, \mathrm{KS}$ (p-values) for Strengths of Glass Fibers

\begin{tabular}{|c|c|c|c|c|c|c|c|c|}
\hline Model & $\alpha$ & $\beta$ & $\gamma$ & $\lambda_{1}$ & $\lambda_{2}$ & W & A & $\begin{array}{c}\text { K-S } \\
\text { (p-value ) }\end{array}$ \\
\hline $\begin{array}{l}\text { CRT } \\
\text { MBIII }\end{array}$ & $\begin{array}{l}3343.164 \\
(4457.274) \\
\end{array}$ & $\begin{array}{l}18.4436 \\
(2.302738) \\
\end{array}$ & $\begin{array}{l}16264.06 \\
(23802.86)\end{array}$ & $\begin{array}{l}0.6463 \\
(0.3772) \\
\end{array}$ & $\begin{array}{l}1.000000 \mathrm{e}-10 \\
(0.8656)\end{array}$ & 0.0390 & 0.2318 & $\begin{array}{l}0.0782 \\
(0.8359) \\
\end{array}$ \\
\hline $\begin{array}{l}\text { TMBI } \\
\text { II }\end{array}$ & $\begin{array}{l}15062.42 \\
(3881.1386)\end{array}$ & $\begin{array}{l}20.0016 \\
(0.7276) \\
\end{array}$ & $\begin{array}{l}82081.20 \\
(8543.3265) \\
\end{array}$ & $\begin{array}{l}0.4698 \\
(0.3181) \\
\end{array}$ & 1 & 0.0807 & 0.4523 & $\begin{array}{l}0.1077 \\
(0.4574) \\
\end{array}$ \\
\hline MBIII & $\begin{array}{l}38353.3724 \\
(4604.0351)\end{array}$ & $\begin{array}{l}20.70762 \\
(0.7271)\end{array}$ & $\begin{array}{l}172677.4910 \\
(6315.7470)\end{array}$ & 1 & 1 & 0.1135 & 0.6271 & $\begin{array}{l}0.1292 \\
(0.2434)\end{array}$ \\
\hline BIII & $\begin{array}{l}3.4417 \\
(0.4505) \\
\end{array}$ & $\begin{array}{l}4.0886 \\
(0.3357) \\
\end{array}$ & 1 & 1 & 1 & 0.9554 & 5.1997 & $\begin{array}{l}0.2462 \\
(0.001) \\
\end{array}$ \\
\hline LL & 1 & $\begin{array}{l}3.4506 \\
(0.3414)\end{array}$ & 1 & 1 & 1 & 0.8045 & 4.4035 & $\begin{array}{l}0.5346 \\
(4.441 \mathrm{e}- \\
16)\end{array}$ \\
\hline
\end{tabular}

Table 5: $-2 \bar{\ell}$, AIC, CAIC, BIC and HQIC for Strengths of Glass Fibers

\begin{tabular}{|l|c|l|l|l|l|}
\hline Model & $-\widehat{2} \hat{\ell}$ & AIC & CAIC & BIC & HQIC \\
\hline CRTMBIII & 19.17072 & 29.17072 & 30.22335 & 39.88639 & 33.38525 \\
\hline TMBIII & 22.18592 & 30.18593 & 30.87558 & 38.75846 & 33.55755 \\
\hline MBIII & 23.82574 & 29.82573 & 30.23251 & 36.25514 & 32.35445 \\
\hline BIII & 73.76724 & 77.76725 & 77.96725 & 82.05352 & 79.45306 \\
\hline LL & 136.9448 & 138.9448 & 139.0104 & 141.088 & 139.7877 \\
\hline
\end{tabular}

From the tables 4 and 5, it is clear that our proposed model is best fitted, with smallest values for all GOFs and maximum $\mathrm{p}$-value. 


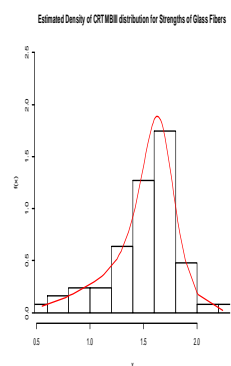

(a)

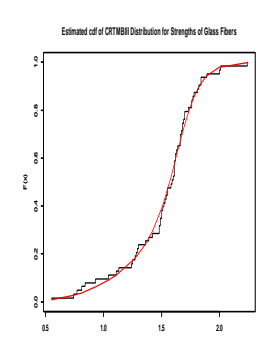

(b)

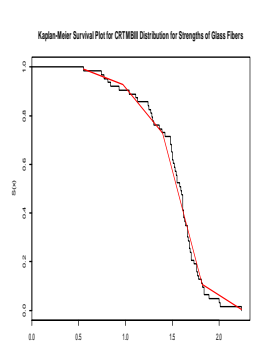

(c)

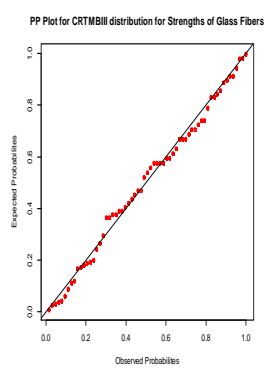

(d)

Figure 4: Fitted (a) pdf, (b) cdf, (c) survival and (d) PP plots for the CRTMBIII distribution to Strengths.

Figure 4 infers that the proposed model is closely fitted to strengths of glass fibers.

\section{CONCLUDING REMARKS}

We propose a very flexible distribution on the basis of the cubic transmuted mapping that is suitable for applications in survival analysis, reliability and actuarial science. The density function of CRTMBIII is symmetrical, right-skewed, left-skewed, exponential, arc, $\mathrm{J}$ and bimodal shaped. The flexible hazard rate of the proposed model can accommodate almost all types of shapes such as unimodal, bimodal, arc, increasing, decreasing, decreasing-increasing-decreasing, inverted bathtub and modified bathtub. We derive the important mathematical properties of the proposed distribution such as survival function, hazard function, reverse hazard function, cumulative hazard function, mills ratio, elasticity, quantile function, moments about the origin, and moments of order statistics, incomplete moments, inequality measures and stress-strength reliability measures. We characterize the proposed distribution via ratio of truncated moments and doubly truncated moment. We address the maximum likelihood estimation for the model parameters. We evaluate the precision of the maximum likelihood estimators via simulation study. We consider an application to real data set to illustrate the flexibility, utility and potentiality of the proposed model. We compute goodness of fit tests for examining the adequacy and competency of the proposed model. We ascertain empirically that the proposed model is suitable for strengths of glass fibers analysis. 


\section{REFERENCES}

[1] Abdul-Moniem, I. B. (2015). Transmuted Burr type III distribution. Journal of Statistics: Advances in Theory and Applications, 14(1), 37-47.

[2] Afify, A.Z., Yousof, H.M. and Nadarajah, S. (2017). The beta transmuted-H family of distributions: properties and applications. Stasistics and its Inference, 10, 505-520.

[3] Ali, A., \& Ahmad, M. (2015). The transmuted modified Burr III distribution. Journal of ISOSS, 1(2), 119-130.

[4] Ali, A., Hasnain, S.A. and Ahmad, M. (2015). Modified Burr III distribution, properties and applications. Pak. J. Stat., 31(6), 697-708.

[5] Alizadeh, M., Merovci, F. and Hamedani, G.G. (2017). Generalized transmuted family of distributions: properties and applications, Hacettepe Journal of Mathematics and Statistics, 46, 4, Doi: 10.15672/HJMS.201610915478.

[6] Al-Kadim, K. A., \& Mohammed, M. H. (2017). The cubic transmuted Weibull distribution. Journal of University of Babylon, 3, 862-876.

[7] Arifa, S., Yab, M. Z., \& Ali, A. (2017). The modified Burr III G family of distributions. Journal of Data Science, 15(1), 41-60.

[8] Bakouch, H., Jamal, F., Chesneau, C., \& Nasir, A. (2017). A new transmuted family of distributions: Properties and estimation with applications. https://hal.archives-ouvertes.fr/hal01570370v3

[9] Bhatti, F.A., Ali, A., and Ahmad, M., (2019). Some recent development of modified Burr III distribution: properties and applications. (Submitted).

[10] Burr, I.W. (1942). Cumulative frequency distributions. Ann. Math. Stat. 13, 215-232.

[11] Glänzel, W. (1987). A characterization theorem based on truncated moments and its application to some distribution families. In Mathematical statistics and probability theory (pp. 75-84). Springer Netherlands

[12] Glänzel, W. A. (1990). Some consequences of a characterization theorem based on truncated moments, Statistics 21 (1990); 613 - 618.

[13] Granzotto, D. C. T., Louzada, F., and Balakrishnan, N. (2017). Cubic rank transmuted distributions: inferential issues and applications. Journal of Statistical Computation and Simulation, 87(14), 2760-2778.

[14] Nofal, Z. M., Afify, A. Z., Yousof, H. M., \& Cordeiro, G. M. (2017). The generalized transmuted-G family of distributions. Communications in Statistics-Theory and Methods, 46(8), 4119-4136.

[15] Rahman, M. M., Al-Zahrani, B., \& Shahbaz, M. Q. (2018). A general transmuted family of distributions. Pakistan Journal of Statistics and Operation Research, 14(2), 451-469.

[16] Rahman, M. M., Al-Zahrani, B., \& Shahbaz, M. Q. (2019). Cubic transmuted Weibull distribution: properties and applications. Annals of Data Science, 6(1), 83-102. 
[17] Riffi, M. I. (2019). Higher rank transmuted families of distributions. IUG Journal of Natural Studies, 27(2), 50-62.

[18] Shaw, W. T., \& Buckley, I. R. (2009). The alchemy of probability distributions: beyond GramCharlier expansions and a skew-kurtotic-normal distribution from a rank transmutation map. arXiv preprint arXiv:0901.0434.

[19] Smith, R. L., \& Naylor, J. (1987). A comparison of maximum likelihood and Bayesian estimators for the three-parameter Weibull distribution. Journal of the Royal Statistical Society: Series C (Applied Statistics), 36(3), 358-369.

[20] Yilmaz, M. (2018). A new distribution family constructed by polynomial rank transmutation. Gazi University Journal of Science, 31(1), 282-294. 